\title{
O papel do enfermeiro no acolhimento e classificação de risco no serviço hospitalar
}

The role of nurses in welcoming and risk classification in the hospital service

El papel de las enfermeras en la acogida y clasificación de riesgos en el servicio hospitalario

Recebido: 09/01/2022 | Revisado: 13/01/2022 | Aceito: 05/02/2022 | Publicado: 13/02/2022

Juliana Souza Andrade

ORCID: https://orcid.org/0000-0003-2267-4586

Faculdades Unidas do Norte de Minas Gerais, Brasil

E-mail: julianasandrade@gmail.com

Sylmara Corrêa Monteiro

ORCID: https://orcid.org/0000-0003-4546-336X Instituto Federal de Educação, Ciência e Tecnologia do Norte de Minas Gerais, Brasil E-mail: scmenfermagem9@gmail.com

Daniel Silva Moraes

ORCID: https://orcid.org/0000-0003-1997-7455

Universidade Estadual de Montes Claros, Brasil E-mail: silvamoraesdaniel@gmail.com

Karla Talita Santos Silva

ORCID: https://orcid.org/0000-0001-5636-1655

Universidade Federal de Minas Gerais, Brasil

E-mail: ktalitass@hotmail.com

Taysa Cristina Cardoso Freitas

ORCID: https://orcid.org/0000-0003-3133-935X

Universidade Estadual de Montes Claros, Brasil

E-mail: cristinacardoso.taysa@gmail.com

Ana Paula de Oliveira Nascimento

ORCID: https://orcid.org/0000-0003-2144-3671

Universidade Federal dos Vales do Jequitinhonha e Mucuri, Brasil

E-mail: anapaulanascimento@ funorte.edu.br.

Edila Alves Moraes Nogueira

ORCID: https://orcid.org/0000-0001-5096-7226

Faculdades Unidas do Norte de Minas Gerais, Brasil

E-mail: edila.enfermagem@yahoo.com.br

Marlete Scremin

ORCID: https://orcid.org/0000-0003-1139-7501 Instituto Federal de Santa Catarina, Brasil E-mail: marlete@ifs.edu.br

Fernando Lucas Freitas Rocha

ORCID: https://orcid.org/0000-0003-2974-4866 Faculdades Unidas do Norte de Minas Gerais, Brasil

E-mail: fernandolucasfreitasroch@gmail.com

Ana Luíza Leobas Moreira Nogueira

ORCID: https://orcid.org/0000-0003-4417-024X

Faculdade de Saúde e Humanidades Ibituruna, Brasil

E-mail: analeobas12@gmail.com

Leidiany Gomes Moreira

ORCID: https://orcid.org/0000-0002-0393-2300

Faculdade de Saúde e Humanidades Ibituruna, Brasil

E-mail: leidanyg@hotmail.com

Gabrielle Terra Dias

ORCID: https://orcid.org/0000-0002-2185-8685

Faculdade de Saúde e Humanidades Ibituruna, Brasil

E-mail: terragabi@hotmail.com

Flabiane Carvalho Cordeiro

ORCID: https://orcid.org/0000-0002-0078-4576

Faculdade de Saúde e Humanidades Ibituruna, Brasil

E-mail: fcordeiro.carvalho@gmail.com

Brenda Cristina Rodrigues de Almeida

ORCID: https://orcid.org/0000-0001-8942-7382

Faculdade de Saúde e Humanidades Ibituruna, Brasil E-mail: brendaroddrigues@gmail.com

Laudileyde Rocha Mota

ORCID: https://orcid.org/0000-0003-3055-1746

Faculdades Santo Agostinho, Brasil

E-mail: laudileyderm@hotmail.com 


\title{
Resumo
}

Introdução: O enfermeiro assume papel de destaque no acolhimento e na classificação de risco nos serviços hospitalares. Objetivo: Conhecer o papel do enfermeiro no acolhimento e na classificação de risco no serviço hospitalar. Metodologia: A pesquisa foi conduzida por meio de revisão integrativa, na qual foram utilizados os bancos de dados secundários LILACS, SCIELO e BDENF, utilizando os descritores "acolhimento; enfermagem em emergência; serviços médicos de emergência e serviço hospitalar de emergência". Resultados: Foram identificados 6 artigos sobre a temática de acordo com os critérios de inclusão. Conclusão: Diante do exposto, foi possível observar uma escassa produção de artigos sobre a temática. Apesar disso, evidenciou-se que a maioria dos artigos abordavam a relevância do papel do enfermeiro no acolhimento na classificação de risco, e a necessidade de qualificação para propiciar decisões de forma segura.

Palavras-chave: Acolhimento; Enfermagem em emergência; Serviços médicos de emergência; Serviço hospitalar de emergência.

\begin{abstract}
Introduction: Nurses play a prominent role in welcoming and classifying risk in hospital services. Objective: To understand the role of nurses in welcoming and in risk classification in the hospital service. Methodology: The research was conducted through an integrative review, in which the secondary databases LILACS, SCIELO and BDENF were used, using the descriptors "reception; emergency nursing; emergency medical services and emergency hospital service". Results: 6 articles on the subject were identified according to the inclusion criteria. Conclusion: Given the above, it was possible to observe a scarce production of articles on the subject. Despite this, it was evident that most articles addressed the relevance of the nurse's role in the reception in the risk classification, and the need for qualification to provide decisions in a safe way.
\end{abstract}

Keywords: Reception; Sick of urgences; Emergency medical services; Hospital service of urgences.

\section{Resumen}

Introduction: Nurses play a prominent role in welcoming and classifying risk in hospital services. Objetivo: Comprender el papel del enfermero en la acogida y en la clasificación de riesgos en el servicio hospitalario. Metodología: La investigación se realizó mediante una revisión integradora, en la que se utilizaron las bases de datos secundarias LILACS, SCIELO y BDENF, utilizando los descriptores "recepción; enfermería de emergencia; servicios médicos de emergencia y servicio hospitalario de emergencia ". Resultados: Se identificaron 6 artículos sobre el tema según los criterios de inclusión. Conclusión: Dado lo anterior, se pudo observar una escasa producción de artículos sobre el tema. A pesar de esto, se evidenció que la mayoría de los artículos abordan la relevancia del rol del enfermero en la recepción en la clasificación de riesgo y la necesidad de calificación para tomar decisiones de manera segura.

Palabras clave: Recepción; Enfermería de urgencias; Servicios médicos de emergencia; Servicio hospitalario de urgencias.

\section{Introdução}

Os serviços hospitalares de urgência são conhecidos como a porta de entrada de um serviço de saúde para os usuários, que oferece um atendimento em tempo hábil e propõe soluções às situações clínicas apresentadas pelos indivíduos, mesmo não exibindo risco a eles (Roncalli et al., 2017). Entretanto, é necessário que a população atendida seja acolhida na chegada ao serviço, pois o acolhimento permite uma visão integral ao estado do paciente, e consequentemente, resulta na organização dos serviços prestados (Guedes et al., 2013).

No atendimento hospitalar, é realizado o acolhimento com classificação de risco (ACCR) que possibilita priorizar os atendimentos segundo a gravidade do paciente, e proporcionar a efetividade no serviço (Feijó et al., 2015). O uso da classificação possibilita oferecer um atendimento humanizado e acolhedor ao indivíduo (Bellucci Júnior et al., 2015).

O ACCR é feito pelo protocolo do Sistema de Triagem de Manchester (STM) que tem o papel de priorizar os pacientes de acordo com a gravidade. Assim, aqueles que possuírem um quadro mais grave, serão atendidos imediatamente e não seguirá o horário de chegada, devido aos riscos apresentados pelo paciente (Rezende et al., 2016). Ou seja, o sistema permite a redução do período de espera em casos que podem gerar danos à vida do indivíduo (Diniz et al., 2014). Já em situações com pouco risco, a espera poderá ser maior e se necessário poderá referenciar-se o usuário para um serviço específico para sua demanda (Bartel et al., 2015).

O STM possui categorias de sinais/sintomas e fluxogramas que são escolhidos de acordo com o relato da queixa do paciente, e posteriormente gera o resultado da classificação. O paciente pode ser classificado em emergente (vermelho) 0 minutos, 
muito urgente (laranja) 10 minutos, urgente (amarelo) 60 minutos, pouco urgente (verde) 120 minutos e não urgente (azul) 240 minutos (Grupo Brasileiro de Classificação De Risco, 2015).

A gravidade do paciente é analisada no momento da classificação de risco, pelo enfermeiro que estabelecerá a prioridade diante do quadro clínico (Souza et al., 2013). Esse profissional tem habilidade para determinar a prioridade necessária naquela circunstância, a partir do seu conhecimento e experiência (Duro et al., 2017).

O enfermeiro irá avaliar o paciente no momento da sua entrada no serviço hospitalar, identificando a queixa, determinando o fluxograma ideal para a situação e então estabelecendo a classificação de risco (HERMIDA et al., 2018). Na classificação é necessário que o paciente e o enfermeiro estejam interligados, para facilitar a identificação da causa e definir o risco de forma adequada (Souza et al., 2015). Além disso, deve-se anotar todas as informações e condutas adotadas em relação ao quadro do paciente (Silva et al., 2013).

Como o enfermeiro apresenta uma função relevante no ato da classificação de risco, é fundamental realizar treinamentos para executar essa tarefa de forma adequada e promover um atendimento de qualidade no serviço de saúde (Pagliotto et al., 2016). Deste modo, a realização da classificação exige que o profissional tenha conhecimento para realizá-la, devido ser um processo minucioso (Oliveira et al., 2013). Assim, objetivou conhecer o papel do enfermeiro no acolhimento e na classificação de risco no serviço hospitalar.

\section{Metodologia}

A pesquisa foi conduzida por meio de revisão integrativa, na qual foram utilizados os bancos de dados do Sistema Latino Americano e do Caribe de Informações em Ciências da Saúde (LILACS), Scientific Eletronic Library Online (SCIELO) e Banco de Dados em Enfermagem (BDENF), utilizando os descritores "acolhimento; enfermagem em emergência; serviços médicos de emergência e serviço hospitalar de emergência".

$\mathrm{Na}$ busca pelos estudos com os descritores "serviço hospitalar de emergência e serviços médicos de emergência" utilizou-se o filtro de assunto principal de triagem. Já no descritor de acolhimento foi selecionado os assuntos principais em acolhimento, humanização da assistência, serviço hospitalar de emergência e assistência à saúde.

Os artigos foram pesquisados no período de junho a setembro de 2021. Como critérios de inclusão para a seleção dos artigos foram adotados os estudos com resumo disponível nas bases de dados com texto completo; publicados no período de 2016 a 2021; artigos em português, inglês, espanhol; e que tratassem da temática abordada neste estudo. Excluíram-se os artigos irrelevantes os quais fugiam do tema; os que não possuíam resumo e texto completo disponível nos bancos de dados; as duplicidades; artigos escritos em outros idiomas que não o português, inglês, espanhol.

Inicialmente foram identificadas 186 referências para esta revisão; destas, 6 artigos foram selecionados por se adequarem ao objetivo proposto. Foram excluídos 180 artigos em razão de duplicidade ou por fugir do tema proposto. Dos artigos selecionados de acordo com a temática e com os critérios de inclusão e exclusão foram distribuídos em um na base SCIELO, dois na base BDENF e três na base LILACS. A partir da seleção dos textos, aplicou-se a leitura flutuante para a organização do corpus de análise, de acordo com o escopo do presente estudo (Figura 1). 
Figura 1. Fluxograma de seleção dos artigos incluídos na revisão.

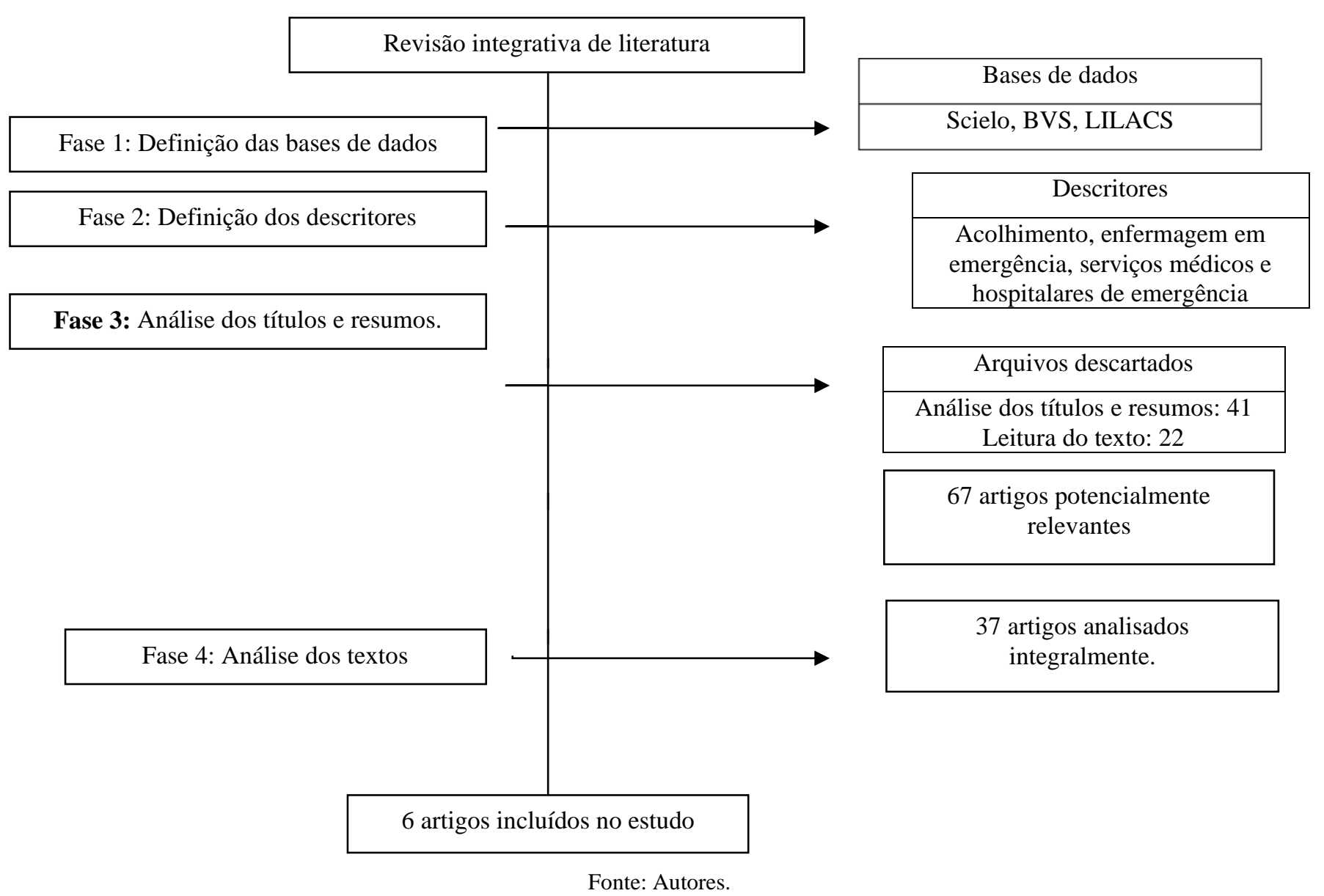

\section{Resultados e Discussão}

O Quadro 1 apresenta os artigos selecionados segundo título, nome do periódico e ano de publicação, autor, objetivos do estudo, síntese das conclusões/recomendações no período de 2016 a 2020. Destacaram-se entre os artigos: dois da Revista Mineira de Enfermagem, dois da Revista Baiana de Enfermagem, um da Revista de Enfermagem da UFSM e um da Revista Investigación y Educación en Enfermería. Quanto à profissão dos autores, dezenove eram enfermeiros, um psicólogo, e um não informou a profissão. As distribuições anuais dos artigos selecionados foram: dois de 2017, dois de 2016 , um de 2015 e um de 2018. 
Quadro 1. Distribuição dos artigos selecionados segundo título, nome do periódico e ano de publicação, autor, objetivos do estudo, síntese das conclusões/recomendações.

\begin{tabular}{|c|c|c|c|c|c|c|}
\hline $\mathrm{N}^{\mathrm{o}}$ & $\begin{array}{c}\text { Título das } \\
\text { Publicações }\end{array}$ & $\begin{array}{c}\text { Nome do } \\
\text { Periódico/Ano de } \\
\text { publicação }\end{array}$ & Autor(es) & Objetivos do estudo & Características do estudo & $\begin{array}{c}\text { Síntese das } \\
\text { Conclusões/Recomendações }\end{array}$ \\
\hline 1 & $\begin{array}{l}\text { Percepção de } \\
\text { enfermeira(o)s } \\
\text { sobre } \\
\text { acolhimento } \\
\text { com } \\
\text { classificação de } \\
\text { risco no serviço } \\
\text { de pronto } \\
\text { atendimento }\end{array}$ & $\begin{array}{l}\text { Revista Baiana de } \\
\text { Enfermagem/2016 }\end{array}$ & $\begin{array}{l}\text { Prudêncio, } \\
\text { C.P.G. Monteiro, } \\
\text { R.A. do N. } \\
\text { Ribeiro, B.C.M.; } \\
\text { Gomes, M.S.M. } \\
\text { Manhães, L.S. P. }\end{array}$ & $\begin{array}{lr}\text { Conhecer a percepção de } \\
\text { enfermeira(o) } & \text { sobre } \\
\text { acolhimento } & \text { com } \\
\text { classificação de } & \text { risco no } \\
\text { serviço de } & \text { pronto } \\
\text { atendimento e } & \text { analisar as } \\
\text { dificuldades } & \text { dessa(e) } \\
\text { enfermeira(o)s } & \text { para } \\
\text { realizarem esse serviço. }\end{array}$ & $\begin{array}{lr}\begin{array}{l}\text { Pesquisa de } \\
\text { abordagem }\end{array} & \text { qualitativa, de } \\
\text { natureza } & \text { descritivo- } \\
\text { exploratória. } & \text { Aplicou-se } \\
\text { questionário } & \text { em } 21 \\
\text { enfermeiras(o)s } & \text { que atuam } \\
\text { no setor de Acolhimento e } \\
\text { Classificação de Risco. }\end{array}$ & $\begin{array}{l}\text { Para a(o) } \\
\text { enfermeira(o)s, a } \\
\text { classificação de risco é um } \\
\text { mecanismo indispensável para } \\
\text { favorecer a otimização da } \\
\text { assistência aos usuários que } \\
\text { apresentam quadros clínicos } \\
\text { específicos de urgência e } \\
\text { emergência, porém não se exclui a } \\
\text { experiência profissional. }\end{array}$ \\
\hline 2 & $\begin{array}{lc}\text { Opinião } & \text { de } \\
\text { enfermeiros } & \\
\text { sobre } & \\
\text { classificação de } \\
\text { risco } & \text { em } \\
\text { serviços } & \text { de } \\
\text { urgência } & \\
\end{array}$ & $\begin{array}{l}\text { Revista Mineira de } \\
\text { Enfermagem/ } \\
2017\end{array}$ & $\begin{array}{l}\text { Duro, C.L.M.; } \\
\text { Lima, M.A.D. da } \\
\text { S. Weber, L.A.F. }\end{array}$ & $\begin{array}{lrrr}\text { Analisar a } & \text { opinião } & \text { dos } \\
\text { enfermeiros } & \text { sobre } & \text { a } \\
\text { atividade de } & \text { CR } & \text { nos } \\
\text { serviços de } & \text { urgência, } \\
\text { indicando } & & \text { suas } \\
\text { potencialidades } & & \mathrm{e} \\
\text { fragilidades. } & & \\
\end{array}$ & 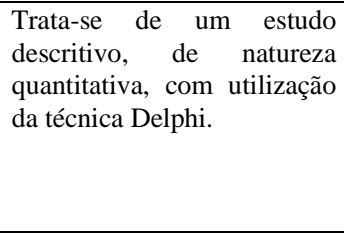 & $\begin{array}{l}\text { Sugerem-se novos estudos sobre a } \\
\text { prática da CR em serviços de } \\
\text { urgência, com vistas a contribuir } \\
\text { para a resolução das fragilidades } \\
\text { identificadas no presente estudo e } \\
\text { qualificar o atendimento à } \\
\text { população. }\end{array}$ \\
\hline 3 & $\begin{array}{l}\text { Processo de } \\
\text { trabalho do } \\
\text { enfermeiro no } \\
\text { acolhimento } \\
\text { com } \\
\text { classificação de } \\
\text { risco }\end{array}$ & $\begin{array}{l}\text { Revista Mineira de } \\
\text { Enfermagem/2016 }\end{array}$ & $\begin{array}{lr}\text { Rates, } & \text { H.F.; } \\
\text { Alves, } & \text { M. } \\
\text { Cavalcante } & \text { R.B. }\end{array}$ & $\begin{array}{l}\text { Descrever o processo de } \\
\text { trabalho do enfermeiro no } \\
\text { setor de Acolhimento com } \\
\text { Classificação de Risco em } \\
\text { uma Unidade de Pronto- } \\
\text { Atendimento. }\end{array}$ & $\begin{array}{l}\text { Realizou-se estudo de caso } \\
\text { de abordagem qualitativa em } \\
\text { uma Unidade de Pronto- } \\
\text { Atendimento.Para } \\
\text { entrevista utilizou-se roteiro } \\
\text { semiestruturado, } \\
\text { entrevistando } \\
\text { enfermeiros. }\end{array}$ & $\begin{array}{l}\text { Ao descrever o processo de trabalho } \\
\text { do enfermeiro no ACCR da UPA, } \\
\text { apurou-se que o mesmo é } \\
\text { reconhecido a partir de seus } \\
\text { elementos (finalidade, objeto, } \\
\text { instrumentos,produtos) } \\
\text { tecnologias. }\end{array}$ \\
\hline 4 & $\begin{array}{l}\text { Protocolo de } \\
\text { Manchester } \\
\text { população } \\
\text { usuária na } \\
\text { classificação de } \\
\text { risco: visão do } \\
\text { enfermeiro }\end{array}$ & $\begin{array}{l}\text { Revista Baiana } \\
\text { Enfermagem } / 2017\end{array}$ & $\begin{array}{l}\text { Roncalli. } \\
\text { Oliveira, } \\
\text { de; Silva, I.C.M. } \\
\text { Brito, } \\
\text { Viegas, S.M. da } \\
\text { F. }\end{array}$ & $\begin{array}{l}\text { Compreender a visão do } \\
\text { enfermeiro sobre a } \\
\text { utilização do protocolo } \\
\text { Manchester e a população } \\
\text { usuária na classificação de } \\
\text { risco de uma Unidade de } \\
\text { Pronto Atendimento. }\end{array}$ & 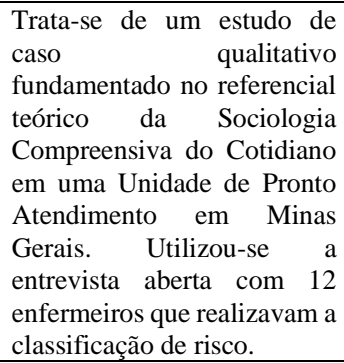 & $\begin{array}{l}\text { Apesar dos desafios para a } \\
\text { concretização da classificação de } \\
\text { risco na Unidade de Pronto } \\
\text { Atendimento como uma estratégia } \\
\text { acolhedora e equânime das } \\
\text { demandas, o protocolo de } \\
\text { Manchester trouxe segurança para a } \\
\text { prática do enfermeiro e qualidade } \\
\text { para a atenção prestada. }\end{array}$ \\
\hline 5 & $\begin{array}{l}\text { Acolhimento e } \\
\text { humanização da } \\
\text { assistência em } \\
\text { pronto-socorro } \\
\text { adulto: } \\
\text { percepções de } \\
\text { enfermeiros }\end{array}$ & $\begin{array}{ll}\text { Revista } & \text { de } \\
\text { Enfermagem } & \text { da } \\
\text { UFSM/2013 } & \end{array}$ & $\begin{array}{l}\text { Neto, A.V. de } \\
\text { L.; Nunes, V.M. } \\
\text { de A.; } \\
\text { FernandeS, R.L.; } \\
\text { Barbosa, I.M.L.; } \\
\text { Carvalho, G.R.P. } \\
\text { de }\end{array}$ & $\begin{array}{l}\text { Descrever as percepções } \\
\text { dos enfermeiros que } \\
\text { trabalham em um pronto- } \\
\text { socorro de atendimento } \\
\text { para pacientes adultos } \\
\text { acerca da humanização e } \\
\text { acolhimento com } \\
\text { classificação de risco. }\end{array}$ & $\begin{array}{l}\text { Trata-se de uma pesquisa } \\
\text { descritiva e exploratória com } \\
\text { abordagem qualitativa, } \\
\text { realizada por meio da coleta } \\
\text { de informações dos } \\
\text { enfermeiros que atuam no } \\
\text { Pronto-Socorro Adulto de } \\
\text { um hospital geral. }\end{array}$ & $\begin{array}{l}\text { A partir dos achados deste estudo, as } \\
\text { práticas institucionais } \\
\text { profissionais poderão ser } \\
\text { aperfeiçoadas para que uma melhor } \\
\text { atenção seja oferecida aos usuários } \\
\text { do serviço. }\end{array}$ \\
\hline 6 & $\begin{array}{l}\text { Percepção do } \\
\text { enfermeiro } \\
\text { sobre a } \\
\text { realização da } \\
\text { classificação do } \\
\text { risco no serviço } \\
\text { de urgências }\end{array}$ & $\begin{array}{l}\text { Revista } \\
\text { Investigación } \quad y \\
\text { Educación en } \\
\text { Enfermería/2014 }\end{array}$ & $\begin{array}{l}\text { Souza, C.C. de; } \\
\text { Diniz, A.S.; } \\
\text { Silva, L. DE } \\
\text { L.T. Mata, } \\
\text { L.R.F. } \\
\text { DA;Chianca,T.C } \\
\text {.M. }\end{array}$ & $\begin{array}{l}\text { Conhecer a percepção dos } \\
\text { enfermeiros sobre a } \\
\text { realização da classificação } \\
\text { do risco no serviço de } \\
\text { urgências. }\end{array}$ & $\begin{array}{l}\text { Trata-se de estudo descritivo } \\
\text { com abordagem qualitativa. } \\
\text { Participaram do estudo } \\
\text { enfermeiros que atuam ou } \\
\text { que já atuaram na } \\
\text { classificação de risco no } \\
\text { local em estudo por um } \\
\text { período mínimo de dois } \\
\text { meses. }\end{array}$ & 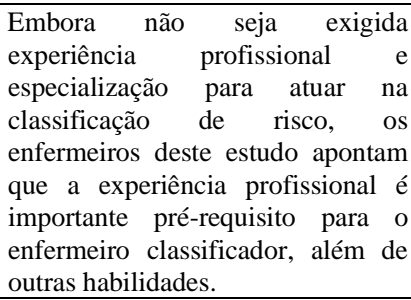 \\
\hline
\end{tabular}

Fonte: Autores.

O enfermeiro exerce uma atividade na classificação de risco considerada como nova e permite se respaldar por meio de protocolos na prática. O STM permite ao enfermeiro identificar e propor soluções de forma eficiente diante do problema apresentado pelo paciente. Além disso, é essencial ter conhecimento para realizar as condutas e promover resultados positivos (Souza et al., 2013; Paglioto et al., 2016).

O enfermeiro deve demonstrar ter domínio das situações e tem a responsabilidade sobre os atendimentos prestados em relação ao processo de organização do serviço de saúde (Bellucci- Júnior, Matsuda, Marcon, 2015). Desse modo, a classificação possibilita que o profissional tenha mais autonomia conforme a participação nos atendimentos (Souza et al., 2014). A 
classificação de risco é uma atividade bem executada pelo enfermeiro, pois ele busca entender a situação do indivíduo de forma holística, analisa o caso, e a partir da sua visão crítica e estabelece o grau de risco e condutas (Prudêncio et al., 2016).

É fundamental que o enfermeiro tenha conhecimento teórico associado à prática, em razão de desencadear uma análise detalhada e conduta em curto prazo no atendimento. Uma vez que a falta de conhecimento pode comprometer os resultados da classificação de risco, causar danos e transtornos ao paciente e sua família (Souza et al., 2013; Hermida et al., 2018).

Esse processo se justifica pela relevância da priorização dos atendimentos em pacientes graves, a fim de evitar agravo do quadro clínico. Outro aspecto importante refere-se a escuta qualificada que permite fornecer informações e medidas eficazes (Rates, Alves, Cavalcante, 2016). Roncalliet et al., (2017) considera que os aspectos como acolher, cuidar, proteger a vida e tratar são desafios vivenciados pelo enfermeiro em seu cotidiano com finalidade de preservar a vida do paciente. Acrescenta-se, que o STM proporciona autoconfiança ao profissional e qualidade na assistência.

Observou-se que o enfermeiro tem a capacidade de classificar o risco do paciente, mas é preciso qualificá-lo quanto a esse processo (Oliveira, 2013). Ressalta-se, que é importante o profissional intensificar a educação continuada junto a carreira profissional, por permitir aprimorar os conhecimentos e fundamentá-los em suas decisões (Caveião et al., 2014).

É relevante que os profissionais recebam capacitação sobre o sistema de Manchester em seu ambiente de trabalho, visando evitar aspectos que possam alterar os resultados da classificação (Malfussi et al., 2018). Os enfermeiros devem receber educação permanente sobre acolhimento e humanização, com o intuito de promover uma classificação correta e atendimento resolutivo (Oliveira, 2013).

O enfermeiro é o profissional que detém maior competência para a realização do acolhimento e classificação de risco. O seu papel refere-se a sua capacidade para identificação de sinais e sintomas por meio da escuta qualificada da queixa principal, o uso do raciocínio clínico e a tomada de decisão rápida e assertiva (Duro, Lima, 2010). Portanto, é necessário ter enfermeiros treinados, e que tenham habilidade para tomar decisões rápidas e adequadas na classificação de risco (Neto et al., 2013). Assim, a capacitação é essencial e possibilita oferecer uma assistência eficiente (Prudêncio et al., 2016).

\section{Conclusão}

A maioria dos artigos abordavam a relevância do papel do enfermeiro no acolhimento e a necessidade de qualificação para propiciar decisões de forma segura. Observou-se que o enfermeiro tem um papel importante no acolhimento e na classificação de risco e precisa ter conhecimento abrangente para analisar os casos dos pacientes, classificando-os criteriosamente e de forma rápida para evitar consequências ao usuário do serviço hospitalar.

Para tanto, é necessário que os enfermeiros sejam capacitados e qualificados para exercer o papel no processo de classificação, a fim de evitar futuros danos aos usuários e aumentar a confiabilidade no uso do protocolo. Assim, sugere-se que sejam realizados novos estudos sobre o assunto, visando esclarecer os papéis e a importância do enfermeiro no acolhimento e classificação de risco.

\section{Referências}

Agência Nacional de Vigilância Sanitária. (2016). Esclarecimentos sobre a regulamentação de industrialização, manipulação, comercialização e registros de insumos, de medicamentos fitoterápicos e de produtos tradicionais fitoterápicos. Informe Técnico nº 007/2016: Anvisa.

Abeso. (2010). Atualização das diretrizes para o tratamento Farmacológico da obesidade e do sobrepeso. ABESO 76: Edição Especial.

Anderson, J. W., Brinkman, V. L., \& Hamilton, C. C. (1992). Weight loss and 2-y follow-up for morbidly obese patients treated with intensive very-low-caloric diet and an education program. Am. J. Clin. Nutr, 56(1), 244-246.

Brasil (2017). Ministério da Saúde. Secretaria de Atenção à Saúde. Departamento de Atenção Básica. Estratégias para o cuidado da pessoa com doença crônica: obesidade / Ministério da Saúde, Secretaria de Atenção à Saúde, Departamento de Atenção Básica. Brasília: Ministério da Saúde. 
Brasil. (2004). Ministério da Saúde. Agência Nacional de Vigilância Sanitária. RDC n48 de 16 de março de 2004. Dispõe sobre o registro de medicamentos fitoterápicos. Diário Oficial, Brasília, 18 de maio.

Conselho Latino Americano sobre Obesidade (2004). Diretrizes Brasileiras de Obesidade $4^{a}$ edição. São Paulo: Conselho Latino Americano Sobre a Obesidade.

Corrêa, E. D. M., Santos, J. M., \& Ribeiro, P. L. B. (2012). Uso de fitoterápicos no tratamento da obesidade: uma revisão bibliográfica. Trabalho de conclusão de curso apresentado ao Centro de Pós Graduação de Nutrição Clínica e Esportiva do Centro de Estudo de Enfermagem e Nutrição da Pontifícia Universidade Católica de Goiás. 2012.

Carvalho, A. C. B, Balbino, E. E., Maciel, A., \& Perfeito, J. P. S. (2008). Situação do registro de medicamentos fitoterápicos no Brasil. Rev. Bras. Farmacog, 18(2), 314-319.

Fetrow, C.W., \& Avila, J.R. (2000). Manual de medicina alternativa. Rio de Janeiro: Guanabara Koogan, 743p.

Gigante, D. P. G., França, G. V. A., Sardinha, L. M. V., Iser, B. P. M., \& Meléndez, G. V. (2011). Variação temporal na prevalência do excesso de peso e obesidade em adultos: Brasil, 2006 a 2009. Revista Brasileira de Epidemiologia, 14(1), 157-165.

Han, L. K., Zheng, Y. N., Yoshikawa, M., Okuda, H., \& kimura, H. (2005). Anti-obesity effects ofchikusetsusaponins isolated from Panax japonicus rhizomes. $B M C, 5(9), 1-10$.

Heckler, A. P. M., Andreazza, Dall'agnol, R. S., Heineck, I., \& Rates, S. M. K. (2005). Estudo Exploratório sobre a Dispensação de Fitoterápicos e Plantas Medicinais em Porto Alegre/RS. Acta Farm, 24(2), 277-283.

IBGE. (2011). Pesquisa de Orçamentos Familiares 2008-2009: análise do consumo alimentar pessoal no Brasil. Rio de Janeiro: Instituto Brasileiro de Geografia e Estatística.

Massi, L., \& Queiroz, S. L. (2015). Iniciação científica: aspectos históricos, organizacionais e formativos da atividade no ensino superior brasileiro [online]. São Paulo: Editora UNESP.

Manenti, A.V. (2010). Plantas Medicinais utilizadas no tratamento da obesidade: uma revisão. 2010. Trabalho de Conclusão de Curso (Nutrição) - Universidade do Extremo Sul Catarinense: UNESC.

Mancini, M. C. (2006). Obesidade: Tratamento. Projeto Diretrizes. Sociedade Brasileira de Endocrinologia e Metabologia.

Mendes K. D. S., Silveira, R, C, C, P., \& Galvão, C. (2018). Revisão integrativa: método de pesquisa para a incorporação de evidências na saúde e na enfermagem. Texto Contexto Enferm, 17(4), 758-764.

Monego, E. T., Peixoto, M. R. G., Jardim, P. C. B. V., Sousa, A. L. L., Braga, V. L. N., \& et al. (1996). Diferentes Terapias no Tratamento da Obesidade em Pacientes Hipertensos. Arq. Bras. Cardiol, 66(6), 343-347.

Moro, C. O., \& Basile, G. (2000). Obesity and medicinal plants. Fitoterapia, 71, 73-82.

Organização Mundial da Saúde. (2000). Obesity: preventing and managing the global epidemic. Report of a WHO consultation on obesity. Geneva, Switzerland: WHO.

Organização Mundial da Saúde. (2009). Obesity and overweight. WHO.

Pinto, C. A., Silva, D. H. S., Bolzani, U. S., Lopes, N. P., \& Epifânio, R. A. (2002). Produtos naturais: atualidade, desafios e perspectivas. Quim.Nova, 25 (5), $45-61$.

Prado, C. N., Neves, D. R. J., Souza, H. D., \& Navarro, F. (2010). O uso de fitoterápicos no tratamento da obesidade. Revista Brasileira de Obesidade, Nutrição e Emagrecimento, 4(9), 14-21.

Rayalam, S., Della-fera, M. A., \& Bail, C. A. (2008). Phytochemicals and regulation of the adipocyte life cycle. J. Nutri. Biochem, 19, 717-726.

Rezende, H. A., \& Cocco, M. I. M. (2002). A utilização de fitoterapia no cotidiano de uma população rural. Rev Esc Enferm USP, 36(3), $282-288$.

Sacramento, H. T. (2000). Fitoterapia nos serviços públicos do Brasil . In: Jornada Paulista de Plantas Medicinais Botucatu. Anais... Botucatu: UNESP, 28.

Senger, A. E. V., Schwanke, C. H. A., \& Gottlieb, M. G.V. (2010). Chá verde (Camellia sinensis) e suas propriedades. Scientia Medica, 20(4), $292-300$.

Tomazzoni, M. I., Negrelle, R. R. B., \& Centa, M. L. (2006). Fitoterapia popular: a busca instrumental enquanto prática terapêutica. Texto Contexto Enferm, 15(1), 115-121.

Trombetta, I. C. (2003). Exercício físico e dieta hipocalórica para o Exercício físico e dieta hipocalórica para o paciente obeso: vantagens e desvantagens. Rev Bras Hipertens, 10(2), 130-133.

Weisheimer, N., Filho, P. F. C., Neves, R. P. C., Sousa, R. M., Pinto, D. S., \& et al. (2015). Fitoterapia como alternativa terapêutica no combate à obesidade. Rev. Ciênc. Saúde Nova Esperança, 13(1), 103-11.

World Health Organization. (2006) Child Growth Standards: length/height-for-age, weight-for-age, weight-for-lenght, weightfor-height and body mass indexfor-age: methods and development. WHO (nonserial publication). Geneva, Switzerland: WHO.

Won, J. W. (2010). Possíveis terapêuticas anti-obesidade da natureza: uma revisão. Elsevier. 
Bartel, T. E. Silva, M. M. Sturbelle, I. C. S. Xavier, S. Q.,Dal Pai, D., \& et al. (2015). Dialogando Sobre Serviços de Saúde a Partir da Implantação do Acolhimento com Avaliação e Classificação de Risco: Relato de Experiência. Revista Baiana de Saúde Pública, 39(1), 164-173.

Bellucci Júnior, J. A., Matsuda, L. M., \& Marcon, S. S. (2015). Análise do fluxo de atendimento de serviço hospitalar de emergência: estudo de caso. Revista Eletrônica de Enfermagem, 17(1), 108-116.

Bellucci Júnior, J. A., Vituri, D. W., Versa, G. L. G. Da S., Furuya, P. S., Vidor, R. C., \& et al. (2015). Acolhimento com classificação de risco em serviço hospitalar de emergência: avaliação do processo de atendimento. Revista Enfermagem UERJ, 23(1), 82-87,

Caveião, C., Hey, A. P., Montezeli, J. H., Barros, A. P. De M. M. \& Sordi, J. A. et al. (2014). Desafios ao enfermeiro na implantação da classificação de risco em unidade mista. Revista de Enfermagem da UFSM, 14(1), 189-196.

Diniz, A. S., Silva, A. P. Da, Souza, C. C. De S., \& Chianca, T. C. M. (2014). Demanda clínica de uma unidade de pronto atendimento, segundo o protocolo de Manchester. Revista Eletrônica de Enfermagem, Goiânia, 16(2), 312-320.

Duro,C. L. M.,Lima, M. A. D.Da S., \& Weber, L. A. F. (2017). Opinião de enfermeiros sobre classificação de risco em serviços de urgência. Revista Mineira De Enfermagem, 21(2), 10-62.

Feijó, V. B. E. R., Cordoni Junior, L., Souza, R. K. T. De S., \& Dias, A. O. (2015). Análise da demanda atendida em unidade de urgência com classificação de risco. Saúde em Debate, Rio de Janeiro, 39(106), 627-636.

Grupo Brasileiro De Classificação De Risco. (2015). Diretrizes para implementação do sistema Manchester de classificação de risco nos pontos de atenção às urgências e emergências: Como implementar o sistema de Manchester de classificação de risco em sua instituição de saúde - Segunda Edição Revisão para o Brasil.

Guedes, M. V. C., Henriques, A. C. P. T., \& Lima, M. M. N. (2013). Acolhimento em um serviço deemergência: percepção dos usuários. Revista Brasileira deEnfermagem, Brasília, 66(1), 31-37.

Hermida, P. M. V., Nascimento, E. R. P. Do, Echevarría-Guanilo, M. E., Brüggemann, O. M., \& Malfussi, L. B. H. (2018). Acolhimento com classificação de risco em unidade de pronto atendimento: estudo avaliativo. Revista da Escola de Enfermagem da USP, 52(2), 105-118.

Malfussi, L. B. H., Bertoncello, K. C. G.Nascimento, E. R. P. Do., Silva, S. G. Da., Hermida, P. M. V., \& et al. (2018). Concordância de um protocolo institucional de avaliação com classificação de risco.Texto \& Contexto Enfermagem, Florianópolis, 27(1), 420-426,

Neto, A. V. De L., Nunes, V. M. De A., Fernandes, R. L, Barbosa, I. M. L., \& Carvalho, G. R. P. De. (2013). Acolhimento e humanização da assistência em pronto-socorro adulto: percepções de enfermeiros. Revista de Enfermagem da UFSM, 3(2), 276 - 286.

Oliveira, G.N.,Vancini-Campanharo, C. R., Okuno, M. F. P., \& Batista, R. E. A. (2013). Cuidados de enfermagem baseados na avaliação e classificação de risco: concordância entre enfermeiros e protocolo institucional. Revista Latino-Americana de Enfermagem, Ribeirão Preto, 21(2), 500-506.

Oliveira, K. K. D. De., Amorim, K. K. P. S., Fernandes, A. P. N. De L., \& Monteiro, A. I. (2013). Impacto da implementação do acolhimento com classificação de risco para o trabalho dos profissionais de uma unidade de pronto atendimento. REME -Revista Mineira de Enfermagem, 17(1), 148-156.

Pagliotto, L. F., Souza, P. B. De., Thomazini, J. O., Ortega, A. B. De A., \& et al. (2016). Classificação de risco em uma unidade de urgência e emergência do interior paulista. CuidArte Enfermagem, 10(2), 148-155.

Prudêncio,C. P.G., Monteiro, R. A. Do N., Ribeiro, B. C. M., Gomes, M. S. M., \& Manhães, L. S. P. (2016). Percepção de enfermeira(o) sobre acolhimento com classificação de risco no serviço de pronto atendimento. Revista Baiana de Enfermagem, Salvador, 30(2), 1-10.

Rates, H. F.,Alves, M., \& Cavalcante, R. B. (2016). O processo de trabalho do enfermeiro no acolhimento com classificação de risco. REME - Revista Mineira de Enfermagem,v. 20(5), 1415-2762.

Rezende,M. R. M., Ercole, F. F., Mattos, S.S.De, \& Donoso, M. T. V. (2016). Protocolo de Manchester em pronto atendimento de hospital escola. Revista Rene, $17(6), 843-849$.

Roncalli, A.A., Oliveira, D. N. De, Silva, I. C. M., Brito, R. F., \& Viegas,S. M. (2017). Protocolo de Manchester e População Usuária na Classificação de Risco: Visão do Enfermeiro. Revista Baiana de Enfermagem, 31(2), 14-51.

Silva, A. P., Diniz, A. S.,Araújo, F. A., \& Souza, C. C. (2013). Presença da queixa de dor em pacientes classificados segundo o protocolo de manchester. Revista de Enfermagem do Centro Oeste Mineiro, 3(1), 507-517.

Souza, C. C. De, Araujo, F. A., \& Chianca, T. C. M. (2015). Literatura Científica sobre a Confiabilidade e Validade do Protocolo do Sistema de Triagem de Manchester (MTS): Uma Revisão Integrativa de Literatura. Revista da Escola de Enfermagem da USP, 49(1), 144-151.

Souza, C. C. De., Diniz, A. S., Silva, L. De L. T., Mata, L. R. F. Da., \& Chianca, T. C. M. (2014). Percepção do enfermeiro sobre a realização da classificação do risco no serviço de urgências. Investigación y Educación En Enfermería, Medellín, 32(1), 78-86.

Souza, C. C., Mata, L. R. F. Da., Carvalho, E. C. De., \& Chianca, T. C. M. (2013). Diagnósticos de enfermagem em pacientes classificados nos níveis I e II de prioridade do Protocolo Manchester. Revista da Escola de Enfermagem da USP, 47(6), 1318-1324. 